\title{
Appendix B: Forecast detail
}

Table BI. Real GDP growth and inflation

\begin{tabular}{|c|c|c|c|c|c|c|c|c|c|c|c|c|}
\hline & \multicolumn{6}{|c|}{ Real GDP growth (per cent) } & \multicolumn{6}{|c|}{ Annual inflation(a) (per cent) } \\
\hline & 2015 & 2016 & 2017 & 2018 & 2019 & $2020-24$ & 2015 & 2016 & 2017 & 2018 & 2019 & $2020-24$ \\
\hline Australia & 2.4 & 2.5 & 2.4 & 2.8 & 2.6 & 3.0 & 1.5 & 1.0 & 1.3 & 2.3 & 2.7 & 3.1 \\
\hline Austria(a) & I.I & 1.5 & 2.5 & 2.0 & 1.5 & 0.8 & 0.8 & 1.0 & 2.1 & 1.4 & 1.5 & 1.6 \\
\hline Belgium(a) & 1.5 & 1.2 & 1.7 & 2.0 & 1.7 & 1.0 & 0.6 & 1.8 & 2.3 & 1.8 & 1.9 & 1.8 \\
\hline Bulgaria(a) & 3.6 & 3.9 & 3.3 & 2.7 & 2.2 & 1.8 & -1.1 & -1.3 & I.I & 1.0 & 1.5 & 1.9 \\
\hline Brazil & -3.8 & -3.6 & 0.5 & 1.0 & 1.9 & 2.6 & 9.0 & 8.7 & 3.4 & 3.2 & 4.8 & 5.9 \\
\hline China & 6.9 & 6.7 & 6.8 & 6.5 & 6.1 & 5.5 & 1.5 & 2.0 & 1.5 & 2.1 & 2.4 & 3.2 \\
\hline Canada & 0.9 & 1.5 & 3.1 & 2.2 & 2.1 & 1.5 & I.I & 1.0 & 1.2 & 1.6 & 1.6 & 1.9 \\
\hline Czech Republic & ic 5.4 & 2.5 & 4.0 & 2.5 & 2.4 & 1.3 & 0.3 & 0.7 & 2.4 & 1.4 & 1.5 & 1.7 \\
\hline Denmark(a) & 1.6 & 1.7 & 1.8 & 1.9 & 2.0 & I.I & 0.2 & 0.0 & 1.3 & 1.5 & 1.5 & 1.8 \\
\hline Estonia(a) & 1.8 & 2.2 & 4.1 & 3.6 & 2.5 & 2.0 & 0.1 & 0.8 & 3.5 & 2.1 & 2.3 & 1.9 \\
\hline Finland(a) & 0.0 & 1.9 & 2.5 & 1.8 & 1.4 & 0.9 & -0.2 & 0.4 & 1.0 & 1.7 & 1.9 & 1.8 \\
\hline France(a) & 1.0 & I.I & 1.7 & 1.7 & 1.6 & 1.4 & 0.1 & 0.3 & 1.2 & 1.2 & 1.5 & 1.6 \\
\hline Germany(a) & 1.5 & 1.9 & 2.1 & 1.8 & 1.7 & 1.3 & 0.1 & 0.4 & 1.7 & 1.5 & 1.5 & 1.5 \\
\hline Greece (a) & -0.3 & 0.0 & I.I & 2.4 & 1.8 & 1.8 & -1.1 & 0.0 & 1.3 & 0.9 & 1.4 & 2.3 \\
\hline Hong Kong & 2.4 & 2.0 & 3.3 & 2.1 & 2.2 & 2.2 & 1.2 & 1.5 & 2.4 & 2.3 & 2.1 & 3.1 \\
\hline Hungary(a) & 3.1 & 1.9 & 3.7 & 3.2 & 2.6 & 1.9 & 0.1 & 0.4 & 2.3 & 2.2 & 2.7 & 3.1 \\
\hline India & 7.5 & 7.9 & 6.8 & 7.6 & 7.3 & 6.9 & 4.9 & 4.9 & 3.1 & 4.8 & 5.5 & 5.0 \\
\hline Indonesia & 4.9 & 5.0 & 4.9 & 5.4 & 4.6 & 4.6 & 6.4 & 3.5 & 4.3 & 5.2 & 5.1 & 4.8 \\
\hline Ireland(a) & 25.5 & 5.1 & 4.0 & 3.6 & 2.7 & 1.8 & 0.0 & -0.2 & 0.3 & 1.0 & 1.5 & 1.7 \\
\hline Italy(a) & 0.9 & I.I & 1.4 & 1.2 & 1.2 & I.I & 0.1 & -0.1 & 1.5 & 1.4 & 2.0 & 1.8 \\
\hline Japan & I.I & 1.0 & 1.5 & 1.0 & 0.9 & 0.8 & 0.4 & -0.4 & 0.0 & 0.6 & 0.9 & 1.4 \\
\hline Lithuania(a) & 2.0 & 2.3 & 3.9 & 3.2 & 2.4 & 0.9 & -0.7 & 0.7 & 3.6 & 1.6 & 1.9 & 1.2 \\
\hline Latvia(a) & 2.7 & 1.5 & 4.0 & 2.5 & 2.4 & 1.7 & 0.2 & 0.1 & 2.7 & I.I & 1.3 & 1.3 \\
\hline Mexico & 2.7 & 2.0 & 2.5 & 2.2 & 2.3 & 2.7 & 2.7 & 2.8 & 5.9 & 4.9 & 3.5 & 3.3 \\
\hline Netherlands(a) & 2.3 & 2.1 & 3.1 & 2.1 & 1.8 & 1.4 & 0.2 & 0.1 & 1.4 & 1.2 & I.I & 1.6 \\
\hline New Zealand & 3.2 & 3.5 & 2.7 & 3.2 & 2.9 & 2.4 & 0.7 & 0.6 & 1.5 & 1.8 & 2.6 & 2.8 \\
\hline Norway & 1.8 & 1.0 & 2.1 & 2.0 & 1.2 & 1.3 & 2.4 & 3.4 & 1.4 & 1.8 & 2.4 & 2.7 \\
\hline Poland & 3.9 & 2.7 & 3.7 & 3.3 & 3.1 & 1.4 & -0.7 & -0.2 & 1.6 & 1.7 & 2.4 & 2.1 \\
\hline Portugal(a) & 1.8 & 1.5 & 2.5 & 1.7 & 1.7 & 1.5 & 0.5 & 0.6 & 1.6 & 1.8 & 1.9 & 1.6 \\
\hline Romania(a) & 4.0 & 4.8 & 5.6 & 4.9 & 3.1 & 1.9 & -0.4 & -1.1 & 0.8 & 2.7 & 3.2 & 2.5 \\
\hline Russia & -2.8 & -0.2 & 1.5 & 1.9 & 2.2 & 1.6 & 15.5 & 7.0 & 3.9 & 3.3 & 4.4 & 4.2 \\
\hline Singapore & 1.9 & 2.0 & 2.8 & 3.2 & 2.1 & 3.2 & -0.5 & -0.5 & 0.7 & 1.4 & 1.9 & 2.8 \\
\hline South Africa & 1.2 & 0.5 & 0.7 & 1.7 & 2.3 & 2.8 & 3.9 & 6.1 & 4.8 & 5.1 & 5.7 & 4.2 \\
\hline S. Korea & 2.8 & 2.8 & 2.9 & 3.2 & 3.4 & 3.8 & 0.7 & 1.0 & 2.1 & 2.1 & 2.0 & 2.1 \\
\hline Slovakia(a) & 3.8 & 3.3 & 3.4 & 4.4 & 2.7 & 1.2 & -0.3 & -0.5 & 1.3 & 2.1 & 1.8 & 1.5 \\
\hline Slovenia(a) & 2.0 & 3.3 & 4.4 & 2.5 & 2.6 & 1.7 & -0.8 & -0.2 & 1.5 & 1.8 & 2.0 & 1.8 \\
\hline Spain (a) & 3.4 & 3.3 & 3.0 & 2.4 & 2.2 & 1.8 & -0.6 & -0.3 & 2.0 & 1.2 & 1.5 & 1.6 \\
\hline Sweden(a) & 4.3 & 3.1 & 2.8 & 2.4 & 2.0 & 1.5 & 0.7 & I.I & 2.0 & 2.0 & 1.8 & 1.9 \\
\hline Switzerland & 1.2 & 1.4 & 0.6 & 1.8 & 2.1 & 2.0 & -0.6 & -0.2 & 0.5 & 0.9 & 1.6 & 1.7 \\
\hline Taiwan & 0.7 & 1.5 & 2.2 & 2.4 & 2.9 & 2.9 & -0.7 & 0.8 & 0.0 & 0.6 & 0.8 & 1.9 \\
\hline Turkey & 5.9 & 3.3 & 5.0 & 3.4 & 3.1 & 2.4 & 7.6 & 7.8 & 10.8 & 9.2 & 8.1 & 5.5 \\
\hline UK(a) & 2.3 & 1.8 & 1.6 & 1.7 & 1.7 & 1.6 & 0.1 & 0.7 & 2.8 & 2.7 & 2.1 & 2.0 \\
\hline US & 2.9 & 1.5 & 2.1 & 2.3 & 2.3 & 2.3 & 0.3 & 1.2 & 1.6 & 2.2 & 2.3 & 2.3 \\
\hline Vietnam & 6.6 & 6.1 & 6.6 & 6.3 & 5.9 & 5.7 & 0.6 & 2.7 & 3.0 & 5.1 & 6.1 & 4.7 \\
\hline Euro Area(a) & 1.9 & 1.8 & 2.1 & 1.9 & 1.7 & 1.4 & 0.0 & 0.2 & 1.6 & 1.4 & 1.6 & 1.6 \\
\hline EU-28(a) & 2.1 & 1.9 & 2.2 & 2.0 & 1.8 & 1.4 & 0.0 & 0.3 & 1.8 & 1.6 & 1.7 & 1.8 \\
\hline OECD & 2.5 & 1.7 & 2.2 & 2.1 & 2.0 & 1.9 & 0.8 & 1.1 & 2.1 & 2.3 & 2.3 & 2.3 \\
\hline World & 3.4 & 3.2 & 3.5 & 3.6 & 3.5 & 3.4 & 3.6 & 3.7 & 3.9 & 4.0 & 3.9 & 3.5 \\
\hline
\end{tabular}

Note: (a) Harmonised consumer price inflation in the EU economies and inflation measured by the consumer expenditure deflator in the rest of the world. 
Table B2. Fiscal balance and government debt

\begin{tabular}{|c|c|c|c|c|c|c|c|c|c|c|c|c|}
\hline & \multicolumn{6}{|c|}{ Fiscal balance (per cent of GDP)(a) } & \multicolumn{6}{|c|}{ Government debt (per cent of GDP, end year)(b) } \\
\hline & 2015 & 2016 & 2017 & 2018 & 2019 & 2024 & 2015 & 2016 & 2017 & 2018 & 2019 & 2024 \\
\hline Australia & -1.5 & -2.0 & -1.2 & -0.3 & 0.0 & -0.8 & 44.0 & 45.1 & 45.7 & 43.9 & 41.8 & 32.6 \\
\hline Austria & -1.1 & -1.5 & -0.6 & -0.6 & -0.5 & -1.4 & 85.5 & 84.6 & 80.6 & 77.0 & 74.3 & 68.6 \\
\hline Belgium & -2.5 & -2.6 & -1.9 & -1.1 & -0.9 & -2.6 & 106.0 & 106.0 & 106.1 & 101.9 & 99.6 & 94.9 \\
\hline Bulgaria & -1.6 & 0.0 & 0.3 & 0.2 & -0.1 & -1.5 & - & - & - & - & - & - \\
\hline Canada & -1.1 & -1.9 & -1.5 & -1.2 & -1.2 & -1.6 & 98.2 & 97.3 & 96.4 & 93.9 & 91.8 & 84.8 \\
\hline Czech Rep. & -0.6 & 0.6 & 0.7 & -0.2 & -0.6 & -1.5 & 39.1 & 36.3 & 37.4 & 35.7 & 34.8 & 35.5 \\
\hline Denmark & -1.3 & -0.9 & -2.1 & -1.9 & -1.5 & -1.6 & 39.6 & 37.8 & 37.2 & 37.7 & 38.5 & 40.3 \\
\hline Estonia & 0.1 & 0.3 & 0.0 & -0.2 & -0.4 & -1.2 & - & - & - & - & - & - \\
\hline Finland & -2.7 & -1.9 & -0.6 & -0.1 & -0.4 & -1.7 & 63.7 & 63.6 & 62.0 & 60.3 & 58.7 & 57.6 \\
\hline France & -3.6 & -3.4 & -3.1 & -2.6 & -2.4 & -2.9 & 96.2 & 96.6 & 98.9 & 98.4 & 97.5 & 95.4 \\
\hline Germany & 0.7 & 0.8 & 0.8 & I.I & 1.0 & -0.9 & 70.9 & 68.1 & 64.0 & 60.4 & 56.8 & 46.3 \\
\hline Greece & -5.9 & 0.7 & -1.1 & -0.9 & -0.3 & -0.2 & 177.7 & 179.4 & 175.4 & 165.1 & 156.6 & 126.9 \\
\hline Hungary & -1.6 & -1.9 & -2.9 & -2.9 & -2.7 & -2.2 & 74.4 & 73.7 & 73.4 & 72.6 & 71.8 & 66.3 \\
\hline Ireland & -2.0 & -0.6 & 0.5 & 0.1 & -0.5 & -1.3 & 78.8 & 75.6 & 73.7 & 70.0 & 67.5 & 62.1 \\
\hline Italy & -2.7 & -2.4 & -2.0 & -1.9 & -1.7 & -2.6 & 132.1 & 132.5 & 134.5 & 132.3 & 129.3 & 120.1 \\
\hline Japan & -3.5 & -4.6 & -4.7 & -4.1 & -3.7 & -4.2 & 218.9 & 221.2 & 225.2 & 225.9 & 225.3 & 215.8 \\
\hline Lithuania & -0.2 & 0.3 & 0.4 & 0.2 & -0.2 & -1.2 & - & - & - & - & - & - \\
\hline Latvia & -1.3 & 0.0 & 0.3 & 0.2 & 0.0 & -0.8 & - & - & - & - & - & - \\
\hline Netherlands & -2.1 & 0.4 & 2.1 & 2.1 & 1.9 & -0.9 & 65.2 & 62.3 & 56.5 & 52.8 & 49.6 & 42.2 \\
\hline Poland & -2.6 & -2.4 & -1.7 & -1.6 & -1.6 & -2.4 & 52.3 & 55.3 & 53.1 & 51.9 & 51.0 & 53.8 \\
\hline Portugal & -4.4 & -2.0 & -2.8 & -2.1 & -1.9 & -1.9 & 129.0 & 130.4 & 129.4 & 127.4 & 125.0 & 115.9 \\
\hline Romania & -0.8 & -3.0 & -3.1 & -3.1 & -2.9 & -1.9 & - & - & - & - & - & - \\
\hline Slovakia & -2.7 & -1.7 & -1.2 & -0.8 & -0.6 & -0.5 & - & - & - & - & - & - \\
\hline Slovenia & -2.9 & -1.8 & -1.0 & -1.0 & -1.2 & -1.8 & - & - & - & - & - & - \\
\hline Spain & -5.1 & -4.5 & -3.2 & -2.2 & -2.0 & -2.2 & 99.8 & 99.4 & 100.1 & 97.5 & 95.3 & 87.8 \\
\hline Sweden & 0.3 & 0.9 & 0.7 & I.I & 0.9 & -0.6 & 43.9 & 41.7 & 39.2 & 36.3 & 33.8 & 29.0 \\
\hline UK & -4.3 & -2.9 & -2.8 & -3.8 & -2.6 & -2.3 & 88.2 & 88.3 & 86.2 & 84.5 & 81.6 & 68.8 \\
\hline US & -4.3 & -5.0 & -4.5 & -4.0 & -3.6 & -2.5 & 103.9 & 105.2 & 105.7 & 105.0 & 103.8 & 95.6 \\
\hline
\end{tabular}

Notes: (a) General government financial balance; Maastricht definition for EU countries. (b) Maastricht definition for EU countries.

Figure BI. World GDP is estimated to have expanded by I. 2 per cent in the second quarter of 2017

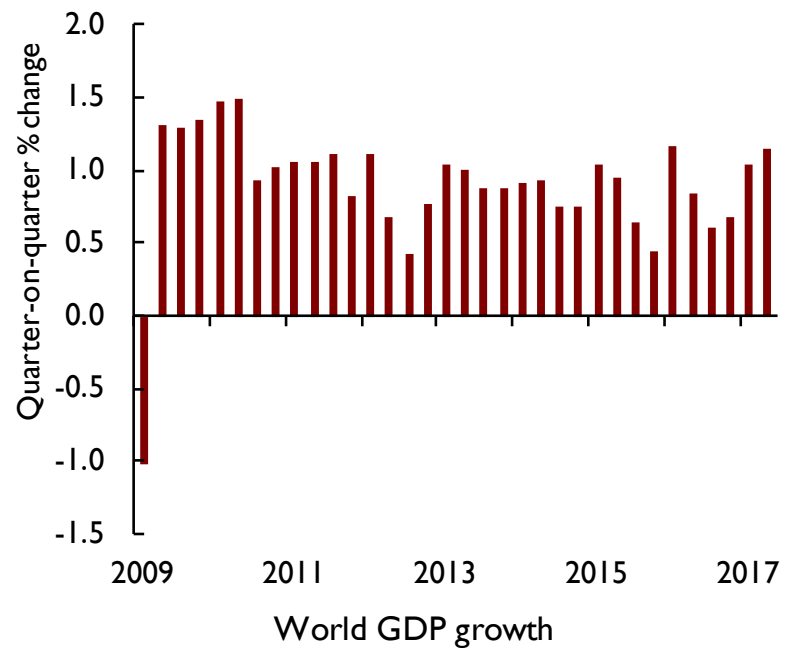

Figure B2. NIESR estimates that world trade grew by 0.9 per cent in 2017Q2

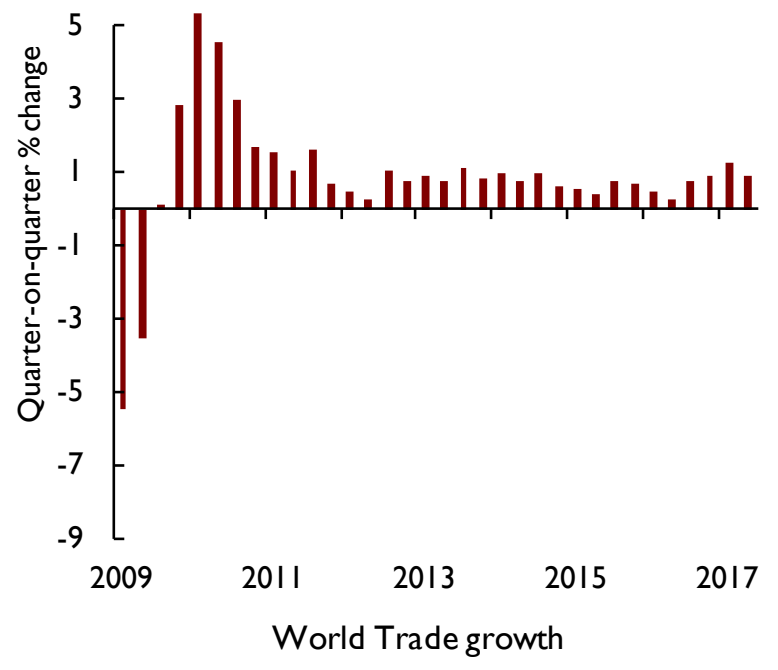


Table B3. Unemployment and current account balance

\begin{tabular}{|c|c|c|c|c|c|c|c|c|c|c|c|c|}
\hline & \multicolumn{6}{|c|}{ Standardised unemployment rate } & \multicolumn{6}{|c|}{ Current account balance (per cent of GDP) } \\
\hline & 2015 & 2016 & 2017 & 2018 & 2019 & $2020-24$ & 2015 & 2016 & 2017 & 2018 & 2019 & $2020-24$ \\
\hline Australia & 6.1 & 5.7 & 5.6 & 5.4 & 5.3 & 5.1 & -4.7 & -2.7 & -2.1 & -2.5 & -2.0 & -2.3 \\
\hline Austria & 5.7 & 6.0 & 5.5 & 5.4 & 5.3 & 4.7 & 1.9 & 2.1 & 0.3 & 0.0 & 0.5 & 0.8 \\
\hline Belgium & 8.5 & 7.8 & 7.4 & 7.3 & 6.9 & 5.4 & -0.2 & 0.1 & -1.3 & -1.4 & -2.0 & -1.2 \\
\hline Bulgaria & 9.1 & 7.6 & 6.2 & 6.0 & 6.6 & 6.9 & 0.0 & 5.4 & 3.5 & 4.4 & 3.9 & 1.7 \\
\hline Canada & 6.9 & 7.0 & 6.4 & 6.3 & 6.4 & 6.6 & -3.4 & -3.3 & -2.8 & -2.4 & -1.6 & -0.4 \\
\hline China & - & - & - & - & - & - & 2.8 & 1.8 & 1.3 & 0.7 & 0.1 & 0.3 \\
\hline Czech Republi & lic 5.1 & 3.9 & 2.9 & 2.3 & 2.1 & 2.1 & 0.2 & I.I & -0.6 & -0.9 & -1.1 & -1.9 \\
\hline Denmark & 6.2 & 6.2 & 5.8 & 5.5 & 5.6 & 5.7 & 9.1 & 7.9 & 7.6 & 6.3 & 5.3 & 6.6 \\
\hline Estonia & 6.2 & 6.7 & 5.9 & 5.4 & 5.7 & 6.1 & 1.9 & 1.9 & 1.3 & 1.9 & 2.2 & 1.7 \\
\hline Finland & 9.3 & 8.9 & 8.7 & 8.2 & 7.9 & 8.2 & -0.6 & -1.1 & -0.5 & -1.5 & -0.5 & 0.6 \\
\hline France & 10.4 & 10.1 & 9.6 & 9.3 & 8.5 & 8.1 & -0.4 & -0.9 & -1.1 & -1.4 & -1.4 & -2.1 \\
\hline Germany & 4.7 & 4.2 & 3.7 & 3.9 & 3.8 & 3.8 & 8.6 & 8.3 & 7.7 & 7.4 & 7.4 & 6.9 \\
\hline Greece & 25.0 & 23.6 & 21.7 & 19.4 & 16.9 & 18.5 & 0.1 & -0.6 & 0.1 & 1.4 & I.I & -1.5 \\
\hline Hungary & 6.8 & 5.1 & 4.3 & 4.4 & 4.5 & 4.1 & 3.5 & 6.2 & 4.5 & 7.4 & 7.3 & 5.9 \\
\hline Ireland & 9.5 & 7.9 & 6.4 & 6.1 & 6.0 & 6.1 & 10.9 & 3.4 & 5.9 & 4.5 & 2.8 & 3.1 \\
\hline Italy & 11.9 & 11.7 & 11.3 & II.I & 10.8 & 10.5 & 1.4 & 2.5 & 2.0 & 1.7 & 2.6 & 3.4 \\
\hline Japan & 3.4 & 3.1 & 2.9 & 2.8 & 3.0 & 3.3 & 3.1 & 3.7 & 3.4 & 3.2 & 3.6 & 4.5 \\
\hline Lithuania & 9.2 & 7.9 & 7.6 & 7.7 & 7.6 & 7.7 & -2.4 & -0.9 & -0.8 & -3.5 & -5.1 & -4.8 \\
\hline Latvia & 9.9 & 9.6 & 8.7 & 8.4 & 8.6 & 8.4 & -0.5 & 1.4 & -1.3 & -0.8 & -0.4 & -0.9 \\
\hline Netherlands & 6.9 & 6.0 & 4.9 & 4.7 & 4.7 & 4.8 & 8.7 & 9.0 & 9.5 & 8.1 & 7.7 & 7.9 \\
\hline Poland & 7.5 & 6.2 & 4.9 & 4.6 & 4.6 & 4.0 & -0.6 & -0.2 & 0.3 & 0.1 & -0.2 & -0.5 \\
\hline Portugal & 12.6 & 11.2 & 9.2 & 8.3 & 8.1 & 9.0 & 0.3 & 0.9 & -0.1 & -1.4 & -1.8 & -1.7 \\
\hline Romania & 6.8 & 5.9 & 5.1 & 5.3 & 5.2 & 5.3 & -1.2 & -2.1 & -3.2 & -2.7 & -2.5 & -2.5 \\
\hline Slovakia & 11.5 & 9.7 & 8.1 & 7.6 & 7.9 & 8.4 & -1.8 & -1.5 & -2.1 & -1.4 & -0.9 & -1.5 \\
\hline Slovenia & 9.0 & 8.0 & 6.9 & 6.7 & 6.6 & 6.7 & 4.4 & 5.2 & 5.0 & 3.8 & 1.9 & -0.8 \\
\hline Spain & 22.0 & 19.6 & 17.4 & 15.3 & 14.0 & 13.4 & I.I & 1.9 & 0.9 & 0.9 & 1.2 & 0.5 \\
\hline Sweden & 7.4 & 6.9 & 6.8 & 6.6 & 6.7 & 7.2 & 4.7 & 4.5 & 4.9 & 5.2 & 5.0 & 4.5 \\
\hline UK & 5.4 & 4.9 & 4.4 & 4.3 & 4.3 & 4.6 & -5.2 & -5.9 & -4.3 & -3.2 & -2.5 & -1.7 \\
\hline US & 5.3 & 4.9 & 4.4 & 4.4 & 4.7 & 5.3 & -2.4 & -2.4 & -2.7 & -3.3 & -3.5 & -3.1 \\
\hline
\end{tabular}

Figure B3. China is not expected to become the world's biggest importer of goods and services in the next 5 years

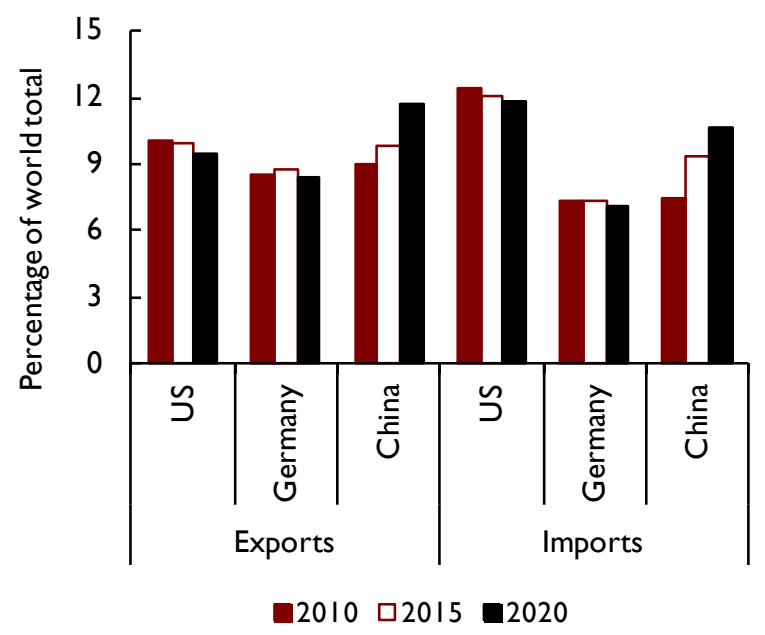

Figure B4. China has overtaken the US as the world's largest economy in 2014

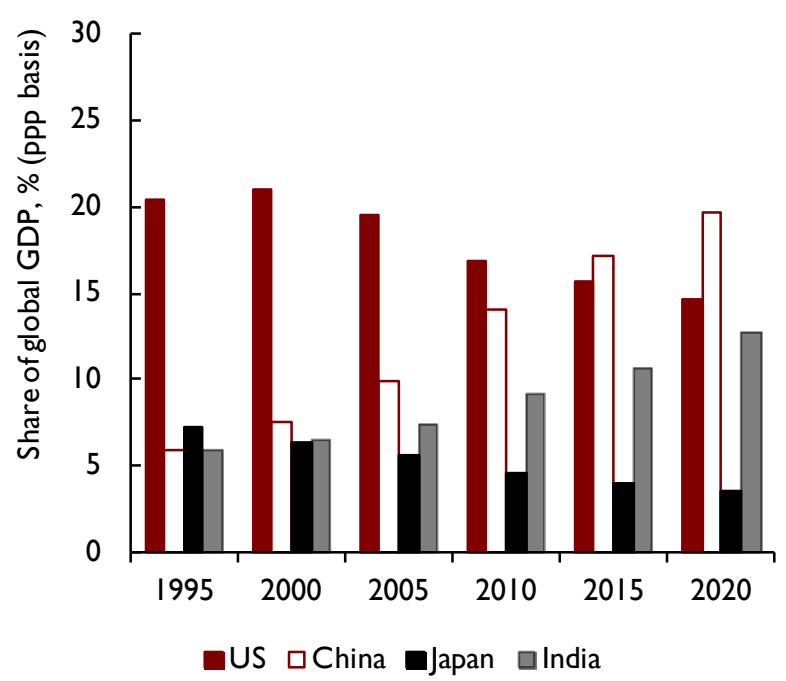


Table B4. United States

Percentage change

\begin{tabular}{|c|c|c|c|c|c|c|c|}
\hline & 2014 & 2015 & 2016 & 2017 & 2018 & 2019 & $\begin{array}{c}\text { Average } \\
2020-24\end{array}$ \\
\hline GDP & 2.6 & 2.9 & 1.5 & 2.1 & 2.3 & 2.3 & 2.3 \\
\hline Consumption & 2.9 & 3.6 & 2.7 & 2.6 & 2.3 & 2.0 & 1.8 \\
\hline Investment : housing & 3.5 & 10.2 & 5.5 & 2.4 & 5.2 & 6.6 & 4.0 \\
\hline : business & 6.9 & 2.3 & -0.6 & 4.0 & 3.8 & 4.0 & 3.0 \\
\hline Government: consumption & -0.5 & 1.3 & 1.0 & 0.0 & 1.4 & 1.6 & 1.8 \\
\hline : investment & -1.4 & 1.6 & -0.2 & 0.6 & 2.8 & 2.5 & 2.0 \\
\hline Stockbuilding(a) & -0.1 & 0.2 & -0.4 & -0.2 & 0.0 & 0.0 & 0.0 \\
\hline Total domestic demand & 2.7 & 3.5 & 1.6 & 2.2 & 2.5 & 2.4 & 2.0 \\
\hline Export volumes & 4.3 & 0.4 & -0.3 & 2.9 & 4.4 & 4.3 & 4.0 \\
\hline Import volumes & 4.5 & 5.0 & 1.3 & 3.5 & 5.4 & 4.5 & 2.2 \\
\hline Average earnings & 2.7 & 2.8 & 1.2 & 1.8 & 3.4 & 3.5 & 3.2 \\
\hline Private consumption deflator & 1.5 & 0.3 & 1.2 & 1.6 & 2.2 & 2.3 & 2.3 \\
\hline RPDI & 3.6 & 4.1 & 1.4 & 1.2 & 1.5 & 1.9 & 1.7 \\
\hline Unemployment, \% & 6.2 & 5.3 & 4.9 & 4.4 & 4.4 & 4.7 & 5.3 \\
\hline General Govt. balance as $\%$ of GDP & -4.9 & -4.3 & -5.0 & -4.5 & -4.0 & -3.6 & -2.9 \\
\hline General Govt. debt as \% of GDP(b) & 103.0 & 103.9 & 105.2 & 105.7 & 105.0 & 103.8 & 99.1 \\
\hline Current account as $\%$ of GDP & -2.1 & -2.4 & -2.4 & -2.7 & -3.3 & -3.5 & -3.1 \\
\hline
\end{tabular}

Note: (a) Change as a percentage of GDP. (b) End-of-year basis.

\begin{tabular}{|c|c|c|c|c|c|c|c|}
\hline & 2014 & 2015 & 2016 & 2017 & 2018 & 2019 & $\begin{array}{c}\text { Average } \\
2020-24\end{array}$ \\
\hline GDP & 2.6 & 0.9 & 1.5 & 3.1 & 2.2 & 2.1 & 1.5 \\
\hline Consumption & 2.7 & 1.9 & 2.3 & 3.5 & 1.9 & 1.5 & 1.1 \\
\hline Investment : housing & 2.7 & 3.8 & 3.0 & 3.4 & 3.0 & 3.3 & 0.9 \\
\hline : business & 3.0 & -11.0 & -8.5 & I.I & 0.2 & 0.0 & 0.4 \\
\hline Government: consumption & 0.8 & 1.5 & 2.0 & 1.4 & 1.8 & 1.7 & 1.6 \\
\hline : investment & -4.2 & 4.5 & I.I & 1.9 & 2.4 & 2.2 & 1.7 \\
\hline Stockbuilding(a) & -0.3 & -0.3 & -0.2 & 0.5 & -0.1 & 0.0 & 0.0 \\
\hline Total domestic demand & 1.7 & 0.2 & 0.9 & 3.4 & 1.7 & 1.5 & 1.1 \\
\hline Export volumes & 5.8 & 3.4 & 1.0 & 2.7 & 3.4 & 4.4 & 2.9 \\
\hline Import volumes & 2.2 & 0.3 & -0.9 & 3.5 & 1.8 & 2.6 & 1.9 \\
\hline Average earnings & 3.2 & 1.8 & 1.8 & 1.9 & 2.5 & 2.8 & 3.1 \\
\hline Private consumption deflator & 1.9 & I.I & 1.0 & 1.2 & 1.6 & 1.6 & 1.9 \\
\hline RPDI & 1.3 & 3.1 & 2.6 & 2.7 & 1.7 & 1.8 & 1.0 \\
\hline Unemployment, \% & 6.9 & 6.9 & 7.0 & 6.4 & 6.3 & 6.4 & 6.6 \\
\hline General Govt. balance as \% of GDP & 0.0 & -1.1 & -1.9 & -1.5 & -1.2 & -1.2 & -1.6 \\
\hline General Govt. debt as \% of GDP(b) & 91.5 & 98.2 & 97.3 & 96.4 & 93.9 & 91.8 & 87.3 \\
\hline Current account as $\%$ of GDP & -2.4 & -3.4 & -3.3 & -2.8 & -2.4 & -1.6 & -0.4 \\
\hline
\end{tabular}

Note: (a) Change as a percentage of GDP. (b) End-of-year basis. 
Table B6. Japan

Percentage change

\begin{tabular}{|c|c|c|c|c|c|c|c|}
\hline & 2014 & 2015 & 2016 & 2017 & 2018 & 2019 & $\begin{array}{c}\text { Average } \\
2020-24\end{array}$ \\
\hline GDP & 0.2 & I.I & 1.0 & 1.5 & 1.0 & 0.9 & 0.8 \\
\hline Consumption & -0.9 & -0.3 & 0.4 & 1.4 & 0.8 & 0.8 & I.I \\
\hline Investment : housing & -4.0 & -1.8 & 5.5 & 4.5 & 1.5 & 1.6 & 3.3 \\
\hline : business & 4.9 & 1.1 & 1.4 & 3.1 & 2.1 & 1.5 & 1.8 \\
\hline Government: consumption & 0.5 & 1.6 & 1.3 & 0.0 & -0.2 & -0.2 & 0.3 \\
\hline : investment & 0.6 & -1.7 & -2.9 & 1.0 & 0.2 & 0.1 & 0.4 \\
\hline Stockbuilding(a) & 0.1 & 0.6 & -0.3 & -0.5 & 0.0 & 0.0 & 0.0 \\
\hline Total domestic demand & 0.3 & 0.8 & 0.4 & 1.0 & 0.8 & 0.7 & I.I \\
\hline Export volumes & 9.3 & 3.0 & I.I & 6.4 & 5.3 & 3.6 & 2.3 \\
\hline Import volumes & 8.2 & 0.7 & -2.3 & 3.9 & 3.9 & 2.5 & 3.8 \\
\hline Average earnings & 0.9 & 0.8 & 1.3 & I.I & 1.8 & 1.8 & 2.2 \\
\hline Private consumption deflator & 2.1 & 0.4 & -0.4 & 0.0 & 0.6 & 0.9 & 1.4 \\
\hline RPDI & -1.7 & 0.8 & 2.1 & 0.8 & 0.7 & 1.0 & 1.3 \\
\hline Unemployment, \% & 3.6 & 3.4 & 3.1 & 2.9 & 2.8 & 3.0 & 3.3 \\
\hline Govt. balance as \% of GDP & -5.4 & -3.5 & -4.6 & -4.7 & -4.1 & -3.7 & -3.8 \\
\hline Govt. debt as \% of GDP(b) & 213.2 & 218.9 & 221.2 & 225.2 & 225.9 & 225.3 & 219.9 \\
\hline Current account as \% of GDP & 0.8 & 3.1 & 3.7 & 3.4 & 3.2 & 3.6 & 4.5 \\
\hline
\end{tabular}

Note: (a) Change as a percentage of GDP. (b) End-of-year basis.

\begin{tabular}{|c|c|c|c|c|c|c|c|}
\hline & 2014 & 2015 & 2016 & 2017 & 2018 & 2019 & $\begin{array}{c}\text { Average } \\
2020-24\end{array}$ \\
\hline GDP & $\mathrm{I} .4$ & 1.9 & 1.8 & 2.1 & 1.9 & 1.7 & 1.4 \\
\hline Consumption & 0.9 & 1.7 & 2.0 & 1.8 & 1.4 & 1.3 & 0.9 \\
\hline Private investment & 2.0 & 3.3 & 3.2 & 4.1 & 2.8 & 2.5 & 2.3 \\
\hline Government : consumption & 0.8 & 1.3 & 1.7 & 1.2 & 1.2 & 1.2 & 1.3 \\
\hline : investment & 0.1 & 2.9 & 1.5 & 1.5 & 1.5 & I.7 & 1.5 \\
\hline Stockbuilding(a) & 0.3 & 0.0 & -0.1 & -0.1 & -0.1 & 0.0 & 0.0 \\
\hline Total domestic demand & 1.3 & 1.9 & 2.0 & 2.0 & 1.6 & 1.5 & 1.3 \\
\hline Export volumes & 4.5 & 6.4 & 3.2 & 4.5 & 4.0 & 3.2 & 2.4 \\
\hline Import volumes & 4.7 & 6.7 & 4.6 & 4.1 & 3.6 & 3.0 & 2.4 \\
\hline Average earnings & 1.2 & 1.3 & 1.6 & 1.4 & 1.7 & 2.0 & 2.6 \\
\hline Harmonised consumer prices & 0.4 & 0.0 & 0.2 & 1.6 & 1.4 & 1.6 & 1.6 \\
\hline RPDI & 0.7 & $\mathrm{I} .4$ & 2.0 & 1.4 & 1.2 & I.7 & 1.2 \\
\hline Unemployment, \% & 11.6 & 10.9 & 10.0 & 9.2 & 8.7 & 8.2 & 8.1 \\
\hline Govt. balance as \% of GDP & -2.6 & -2.1 & -1.5 & -1.0 & -0.7 & -0.7 & -1.4 \\
\hline Govt. debt as \% of GDP(b) & 92.7 & 91.1 & 90.0 & 87.8 & 84.9 & 82.4 & 77.5 \\
\hline Current account as \% of GDP & 2.4 & 3.2 & 3.3 & 2.7 & 2.5 & 2.5 & 2.3 \\
\hline
\end{tabular}

Note: (a) Change as a percentage of GDP. (b) End-of-year basis; Maastricht definition. 
Table B8. Germany

Percentage change

\begin{tabular}{|c|c|c|c|c|c|c|c|}
\hline & 2014 & 2015 & 2016 & 2017 & 2018 & 2019 & $\begin{array}{c}\text { Average } \\
2020-24\end{array}$ \\
\hline GDP & 1.9 & 1.5 & 1.9 & 2.1 & 1.8 & 1.7 & 1.3 \\
\hline Consumption & 1.0 & 1.6 & 1.9 & 2.1 & 1.5 & 1.3 & 0.5 \\
\hline Investment : housing & 3.1 & -1.2 & 3.8 & 5.5 & 2.2 & 1.5 & 1.7 \\
\hline : business & 4.8 & 1.4 & 2.5 & 2.9 & 2.1 & 1.5 & 1.0 \\
\hline Government: consumption & 1.5 & 2.9 & 3.7 & 1.5 & 1.0 & 0.8 & 0.8 \\
\hline : investment & -1.2 & 4.5 & 2.6 & 4.1 & 1.8 & 1.6 & 0.9 \\
\hline Stockbuilding(a) & -0.3 & -0.3 & -0.1 & -0.2 & 0.0 & 0.0 & 0.0 \\
\hline Total domestic demand & 1.3 & 1.5 & 2.4 & 2.2 & 1.6 & 1.3 & 0.7 \\
\hline Export volumes & 4.5 & 4.7 & 2.4 & 3.9 & 4.2 & 3.7 & 2.7 \\
\hline Import volumes & 3.5 & 5.2 & 3.8 & 4.4 & 4.1 & 3.0 & 1.7 \\
\hline Average earnings & 2.5 & 3.0 & 2.9 & 2.0 & 2.2 & 2.1 & 2.4 \\
\hline Harmonised consumer prices & 0.8 & 0.1 & 0.4 & 1.7 & 1.5 & 1.5 & 1.5 \\
\hline RPDI & 1.5 & 1.9 & 2.1 & 1.8 & 0.6 & 1.0 & 0.6 \\
\hline Unemployment, \% & 5.0 & 4.7 & 4.2 & 3.7 & 3.9 & 3.8 & 3.8 \\
\hline Govt. balance as \% of GDP & 0.3 & 0.7 & 0.8 & 0.8 & I.I & 1.0 & -0.1 \\
\hline Govt. debt as \% of GDP(b) & 74.6 & 70.9 & 68.1 & 64.0 & 60.4 & 56.8 & 49.7 \\
\hline Current account as $\%$ of GDP & 7.5 & 8.6 & 8.3 & 7.7 & 7.4 & 7.4 & 6.9 \\
\hline
\end{tabular}

Note: (a) Change as a percentage of GDP. (b) End-of-year basis; Maastricht definition.

Table B9. France

\begin{tabular}{|c|c|c|c|c|c|c|c|}
\hline & 2014 & 2015 & 2016 & 2017 & 2018 & 2019 & $\begin{array}{c}\text { Average } \\
2020-24\end{array}$ \\
\hline GDP & 1.0 & 1.0 & I.I & 1.7 & 1.7 & 1.6 & 1.4 \\
\hline Consumption & 0.7 & 1.3 & 2.1 & I.I & 1.6 & 1.7 & 1.2 \\
\hline Investment : housing & -3.0 & -2.1 & 2.4 & 4.9 & 4.0 & 5.1 & 6.6 \\
\hline : business & 2.9 & 3.1 & 3.6 & 4.2 & 4.1 & 3.6 & 2.2 \\
\hline Government: consumption & 1.3 & 1.1 & 1.2 & 1.2 & 1.0 & 1.3 & 1.8 \\
\hline : investment & -5.4 & -3.0 & -0.1 & -1.3 & 0.9 & 1.3 & 1.9 \\
\hline Stockbuilding(a) & 0.7 & 0.3 & -0.1 & 0.3 & -0.3 & 0.0 & 0.0 \\
\hline Total domestic demand & 1.5 & 1.5 & 1.9 & 1.9 & 1.6 & 2.0 & 1.8 \\
\hline Export volumes & 3.4 & 4.0 & 1.9 & 3.3 & 5.3 & 4.0 & 2.5 \\
\hline Import volumes & 4.8 & 5.5 & 4.2 & 3.8 & 4.3 & 4.8 & 3.3 \\
\hline Average earnings & 1.2 & 0.5 & 1.9 & 1.9 & 1.4 & 2.0 & 3.1 \\
\hline Harmonised consumer prices & 0.6 & 0.1 & 0.3 & 1.2 & 1.2 & 1.5 & 1.6 \\
\hline RPDI & 0.7 & 1.2 & 2.0 & 1.0 & 1.6 & 2.0 & 1.6 \\
\hline Unemployment, \% & 10.3 & 10.4 & 10.1 & 9.6 & 9.3 & 8.5 & 8.1 \\
\hline Govt. balance as $\%$ of GDP & -3.9 & -3.6 & -3.4 & -3.1 & -2.6 & -2.4 & -2.7 \\
\hline Govt. debt as \% of GDP(b) & 95.2 & 96.2 & 96.6 & 98.9 & 98.4 & 97.5 & 95.8 \\
\hline Current account as $\%$ of GDP & -1.3 & -0.4 & -0.9 & -1.1 & -1.4 & -1.4 & -2.1 \\
\hline
\end{tabular}

Note: (a) Change as a percentage of GDP. (b) End-of-year basis; Maastricht definition. 
Table BI0. Italy

Percentage change

\begin{tabular}{|c|c|c|c|c|c|c|c|}
\hline & 2014 & 2015 & 2016 & 2017 & 2018 & 2019 & $\begin{array}{c}\text { Average } \\
2020-24\end{array}$ \\
\hline GDP & 0.2 & 0.9 & I.I & 1.4 & 1.2 & 1.2 & I.I \\
\hline Consumption & 0.2 & 2.0 & 1.5 & 1.3 & 0.7 & 0.3 & 0.2 \\
\hline Investment : housing & -6.7 & -1.8 & 2.8 & 1.5 & 0.5 & 2.1 & 3.6 \\
\hline : business & 0.2 & 3.9 & 1.6 & 0.7 & 0.1 & 2.2 & 3.6 \\
\hline Government : consumption & -0.7 & -0.6 & 0.5 & 0.9 & 0.9 & 0.7 & 0.8 \\
\hline : investment & -3.5 & -1.5 & 9.2 & 2.3 & 0.9 & 0.5 & 0.8 \\
\hline Stockbuilding(a) & 0.7 & 0.0 & -0.3 & 0.2 & 0.1 & 0.0 & 0.0 \\
\hline Total domestic demand & 0.3 & 1.4 & 1.2 & 1.3 & 0.8 & 0.7 & 0.9 \\
\hline Export volumes & 2.4 & 4.2 & 2.6 & 5.0 & 3.2 & 2.6 & 2.2 \\
\hline Import volumes & 3.1 & 6.6 & 3.3 & 5.5 & 2.2 & 0.9 & 1.7 \\
\hline Average earnings & 0.4 & 0.6 & 0.4 & 1.0 & 0.4 & 1.3 & 1.8 \\
\hline Harmonised consumer prices & 0.2 & 0.1 & -0.1 & 1.5 & 1.4 & 2.0 & 1.8 \\
\hline RPDI & 0.5 & 0.8 & 1.6 & 0.6 & 0.5 & 0.8 & 0.4 \\
\hline Unemployment, \% & 12.6 & 11.9 & 11.7 & 11.3 & 11.1 & 10.8 & 10.5 \\
\hline Govt. balance as \% of GDP & -3.0 & -2.7 & -2.4 & -2.0 & -1.9 & -1.7 & -2.3 \\
\hline Govt. debt as \% of GDP(b) & 131.7 & $132 . \mid$ & 132.5 & 134.5 & 132.3 & 129.3 & 123.3 \\
\hline Current account as \% of GDP & 1.8 & 1.4 & 2.5 & 2.0 & 1.7 & 2.6 & 3.4 \\
\hline
\end{tabular}

Note: (a) Change as a percentage of GDP. (b) End-of-year basis; Maastricht definition.

Table BI I. Spain

Percentage change

\begin{tabular}{|c|c|c|c|c|c|c|c|}
\hline & 2014 & 2015 & 2016 & 2017 & 2018 & 2019 & $\begin{array}{c}\text { Average } \\
2020-24\end{array}$ \\
\hline GDP & 1.4 & 3.4 & 3.3 & 3.0 & 2.4 & 2.2 & 1.8 \\
\hline Consumption & 1.5 & 3.0 & 3.0 & 2.4 & 1.9 & 1.8 & 1.4 \\
\hline Investment : housing & 6.2 & 3.1 & 3.7 & 6.5 & 2.4 & 2.1 & 2.8 \\
\hline : business & -0.5 & 4.0 & 6.0 & 3.7 & 3.7 & 2.2 & 2.1 \\
\hline Government: consumption & -0.3 & 2.1 & 0.8 & I.I & 1.4 & 1.9 & 2.0 \\
\hline : investment & 12.8 & 16.1 & -1.5 & 2.8 & 1.9 & 3.0 & 2.0 \\
\hline Stockbuilding(a) & 0.2 & 0.4 & 0.0 & -0.1 & 0.0 & 0.0 & 0.0 \\
\hline Total domestic demand & 2.0 & 4.0 & 2.6 & 2.5 & 2.0 & 2.0 & 1.7 \\
\hline Export volumes & 4.3 & 4.2 & 4.8 & 5.8 & 4.7 & 4. 1 & 3.0 \\
\hline Import volumes & 6.6 & 5.9 & 2.7 & 4.6 & 3.7 & 3.7 & 3.0 \\
\hline Average earnings & 0.0 & 1.3 & 0.3 & 0.7 & 2.0 & 2.0 & 2.8 \\
\hline Harmonised consumer prices & -0.2 & -0.6 & -0.3 & 2.0 & 1.2 & 1.5 & 1.6 \\
\hline RPDI & 0.9 & 1.9 & 2.6 & 1.2 & 2.2 & 2.3 & 1.5 \\
\hline Unemployment, \% & 24.5 & 22.0 & 19.6 & 17.4 & 15.3 & 14.0 & 13.4 \\
\hline Govt. balance as \% of GDP & -6.0 & -5.1 & -4.5 & -3.2 & -2.2 & -2.0 & -2.2 \\
\hline Govt. debt as $\%$ of GDP(b) & 100.4 & 99.8 & 99.4 & 100.1 & 97.5 & 95.3 & 90.4 \\
\hline Current account as $\%$ of GDP & 1.0 & I.I & 1.9 & 0.9 & 0.9 & 1.2 & 0.5 \\
\hline
\end{tabular}

Note: (a) Change as a percentage of GDP. (b) End-of-year basis; Maastricht definition. 